\title{
Dumb-bell neurinoma of the hypoglossal nerve ${ }^{1}$
}

\author{
A.D. BARTAL, M. M. DJAldetti, E. M. MANDEL, AND M. A. LeRner \\ From the Department of Surgical Neurology, Ichilov Hospital, Tel-Aviv Medical Center, and Departments of \\ Internal Medicine ' $B$ ' and Radiology, Hasharon Hospital, Kupat Holim, Petah Tiqva Municipality, and Tel- \\ Aviv University Medical School, Israel
}

SUMMARY A unique case of dumb-bell neurinoma of the hypoglossal nerve is presented. The importance of hemiatrophy of the tongue is stressed for early diagnosis of the lesion. The myelocisternogram is the single most important contrast study confirming the intracranial extension of the tumour. Total dissection of the tumour capsule may not be feasible in all cases.

Neurinomas of the hypoglossal nerve are rare and have attracted sporadic attention. In 1967, Ignelzi and Bucy reported on a patient and reviewed nine additional cases of intracranial hypoglossal neurofibromas from the literature. The following case appears to be unique because of the intra- and extracranial growth of the tumour. The appearance almost eight years previously of hemiatrophic deviation and fibrillations of the tongue should have been recognized as an important diagnostic sign.

\section{CASE REPORT}

This 36 year old male was admitted urgently to the Neurosurgical Department of Tel-Aviv Medical Center on 14 March 1971, because of severe headaches, vomiting, dizziness, blurred vision, bilateral papilloedema and imbalance of gait. He had been admitted to another hospital three weeks earlier with a diagnosis of viral encephalitis or hypertensive encephalopathy.

Eight years before his admission atrophy of the left side of the tongue was noted on a routine examination for sore throat. One year later he started complaining of headaches, and blood pressure of 200/140 mm $\mathrm{Hg}$ was found. He was treated with reserpine, methyldopa, chlordiazepoxide, and frusemide. Repeated examinations during the succeeding years revealed no cause for the vascular hypertension.

He was a physically fit, athletically built man with a left torticollis and marked protective rigidity of the neck. He walked on a broad base and tended to veer

1 This work was supported by a research grant from the Rofei Holim Foundation, New York, to the Judith Herman (Leibovits) Neurosurgical Brain Cancer Research Unit. to the left and the stance was unsteady, worse with eyes closed. There was tenderness in the left retroauricular region and a mass was felt in the neck under the left maxillary angle. Below the bulging of tonsilar recess a hard non-pulsatile mass was felt oाu bimanual palpation.

A fine horizontal nystagmus was noted on lef lateral gaze with the slow component to the lefo The pupils were normal. There was bilateral papil oedema with markedly engorged veins and numerous haemorrhages, punctate exudates, and arteriovenous nipping of the retinal vessels. No involvement of cranial nerves V, VI, VII, VIII, and IX was foun but a mild hoarseness was noted. Asymmetry of the shoulder girdle with drooping of the left side was seen but there was no noticeable atrophy of the left shoulder muscle group.

Movements of the upper and lower extremities, muscle tone, and the reflexes were symmetrical.

The blood pressure was $160 / 100 \mathrm{mmHg}$, the pulse $\bar{\partial}$ rate 80 per minute, and the electrocardiogram showed flattened $\mathrm{T}$-waves, signs of left heart hypertrophy, left axis deviation, and a suspicion of a posterior wall ischaemic lesion. The electroencephalograph (EEG) showed 2 to $3 \mathrm{~Hz}$ slow waves, predominantly in the right frontal region, over a background of a generally disturbed record.

RADIOGRAPHIC INVESTIGATIONS In the axial projection of the skull the medial edge of the cervical mass produced an inward bulge of the pharyngeal $\frac{7}{0}$ wall. The bony abnormalities were fully appreciated only by laminography: the left occipital condyle, $N$ in both anteroposterior (Fig. 1) and lateral projec- $N$ tions (Fig. 2a and b) was found to be widely cavitated $\mathcal{N}$ as a result of concentric widening of the hypoglossal 


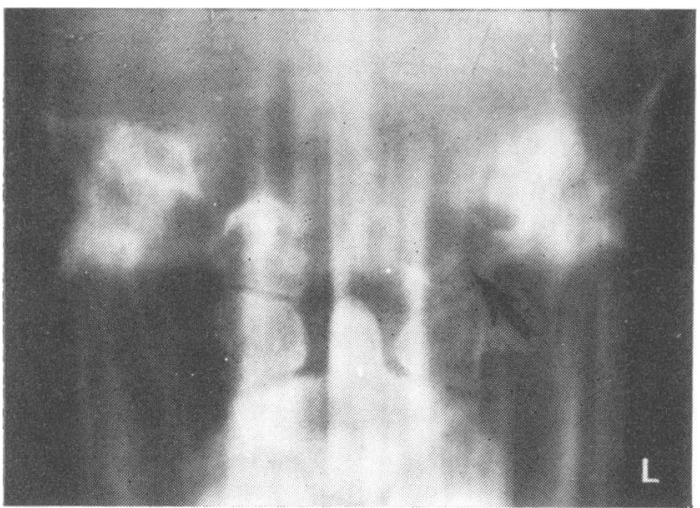

FIG. 1. Anteroposterior tomography shows the defect of the bony structure of the left occipital condyle (arrow).
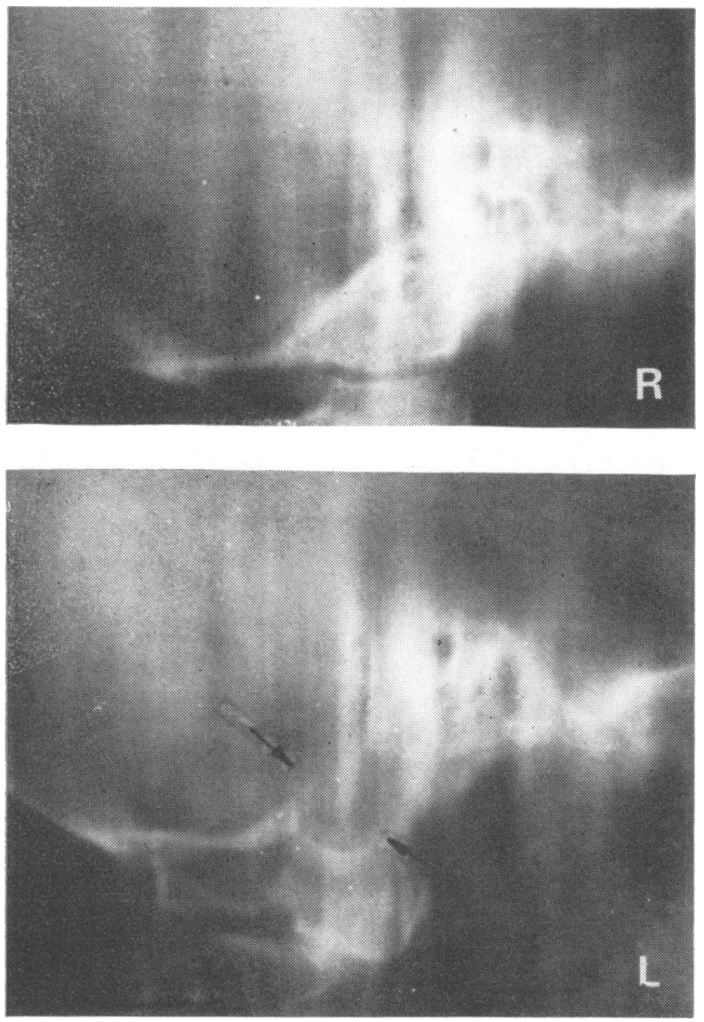

FIG. 2. (a, top) Lateral tomography, base of skull, shows a normal right lateral tomogram for comparison. (b, bottom) The 'blown out' hypoglossal canal excavates completely the left occipital condyle and erupts into the jugular foramen.

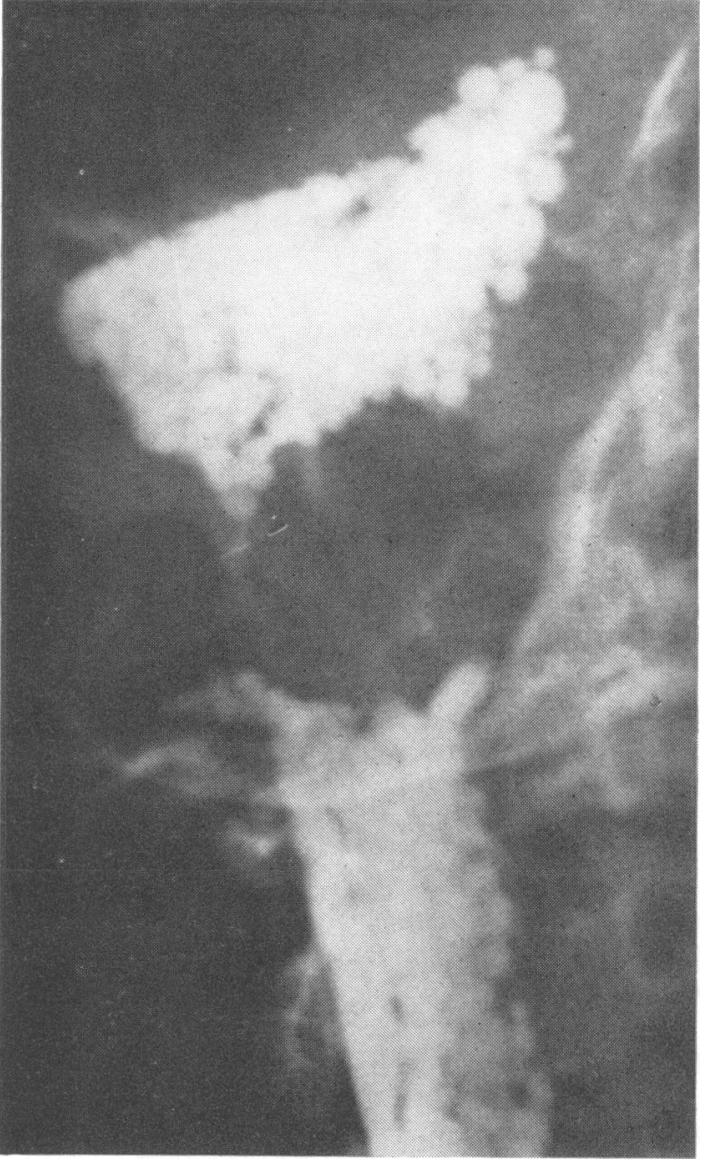

FIG. 3. Cisternomyelogram delineating the retroclinoid component of the tumour.

canal. Hypoglossal neurinoma was diagnosed on this basis.

The intracranial extension of the lesion was delineated by the cisternomyelogram (Fig. 3), while the cervical component of the neurinoma displaced the internal and external carotid arteries forward (Fig. 4); only a fine capillary blush was apparent. The left vertebral artery was displaced upwards and posteriorly.

OPERATION At left suboccipital craniectomy under endotracheal anaesthesia the dura mater was found to be extremely tense. After incision of the dura mater and elevation of the left cerebellar lobe, a grey-white tumour mass was uncovered. Blood vessels and cranial nerves IX, X, XI were seen tensely stretched over it. Dissection was carried out with the use of optic magnification. Firm adhesions to the 


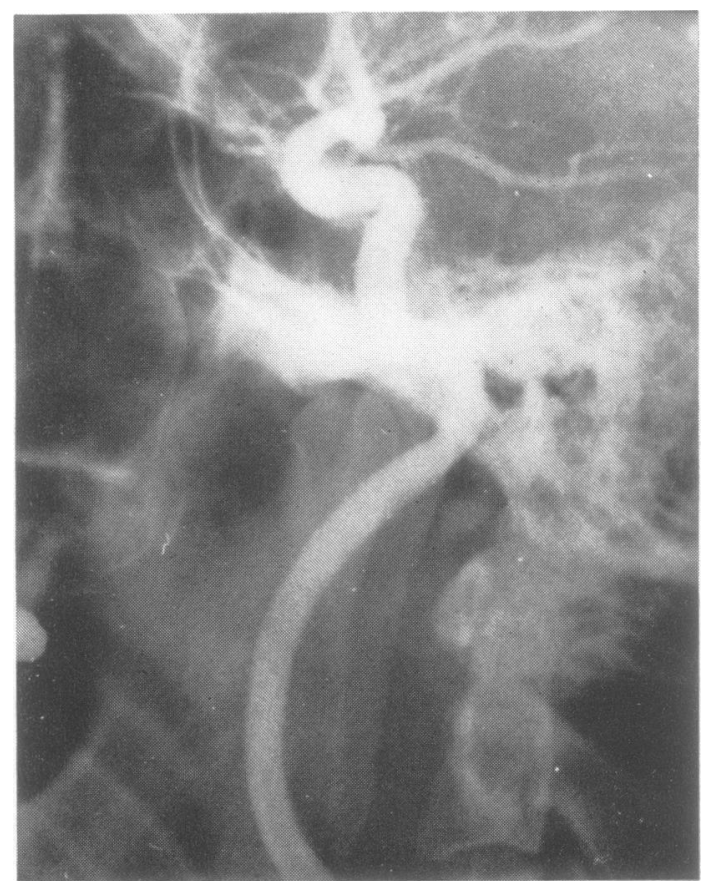

FIG. 4. Selective internal carotid angiogram. Anterior bowing of the artery over cervical component of tumour.

left anterolateral medulla were encountered, and although the major part of the capsule was excised, the medial part, adherent to the medulla, was left behind. Tracheostomy was performed before extubation and was closed after two days.

A month later dissection of the tumour in the neck was performed under endotracheal anaesthesia. The anterior border of the sternocleidomastoid muscle was exposed and a $7 \mathrm{~cm}$ tumour with a smooth glistening capsule was dissected out up to the base of the skull. Histological examination of both tumours was typical of an Antoni type I neurilemomma.

During the postoperative period the suboccipital craniectomy bulged and repeated lumbar puncture was necessary. To obviate postoperative hydrocephalus, an atrioventricular shunt was introduced and the patient's condition improved dramatically. Neither the hemiatrophy of the tongue with fasciculations nor the hoarseness improved but gait and stance became much more stable and papilloedema resolved. Blood pressure remained elevated (160/110 $\mathrm{mmHg}$ ) with occasional bouts of hypertension reaching $200 \mathrm{mmHg}$ systolic. He resumed his previous occupation and when seen in our outpatient department a year later he was found to be well.

\section{DISCUSSION}

In their discussion of 10 cases Ignelzi and Bucy (1967) have reviewed comprehensively the clinical presentation of the tumour. Most of the cases were brought to the physician because of symptoms due to compression of the brain-stem or increased intracranial pressure rather than those related to the hypoglossal nerve, yet the latter antedated other symptoms from five months up to 10 years, on average $3 \cdot 1$ years. In our case hemiatrophy was noticed eight years before admission. Despite advanced hemiatrophy and fasciculations of the tongue most patients knew of it only after its discovery by the examining physician.

Vague sensory disturbances, wasting of shoulder girdle muscles, hoarseness, and nystagmus have been noted (Scott and Wycis, 1949; Raney, Raney, and Bayless, 1954; McGrew, 1955; Morelli, 1966; Ignelzi and Bucy, 1967). Often degenerative neurological disease such as syringobulbia, amyotrophic lateral sclerosis (bul- in bar form), and multiple sclerosis were treated $\mathrm{d}$ erroneously for many years until the diagnosiso was made when signs of increasing intracraniaf pressure developed (McGrew, 1955). Cerebella symptoms, ipsi- and contralateral pyramidab tract dysfunction, and papilloedema resulting from obstructive hydrocephalus were seen in most advanced cases.

Ignelzi and Bucy (1967) stated that benign tumours (neurofibromas and meningiomas) in the proximity of the foramen magnum showed surprisingly few radiological changes, in marked contrast with the mixed group of tumours in this area.

A composite picture of the individual bony and myelographic findings emerges from the case reports of hypoglossal neurinomas. The present case includes all of them.

The ideal projections for proper evaluation of the hypoglossal canal by tomography-routine skull projections being inadequate for the purpose-have been worked out by Kirdani (1967) and norms established. Valvassori and Kirdani (1967) presented 14 cases of hypoglossal canal erosion, five of which were due to hypoglossal neurinomas, and emphasized again the indispensability of tomography in the appropriate clinical setting. Excavation of the canal from 
within, as the tumour grows within the canal, differentiates neurinoma from extrinsic neoplastic (chemodectoma, chordoma, meningioma, lymphoma, and metastasis) and primary bone diseases such as Paget's disease and osteomyelitis.

Myelography and angiography, as in this case, enable a planned, safe approach to the removal of the tumour. They also serve to exclude risky vascular tumours such as angiofibroma and chemodectoma which also originate in the intracranial or parapharyngeal region.

The high surgical mortality, greater than $60 \%$, is believed to be due primarily to respiratory dysfunction. In view of this and in order to avoid a possible lesion of the lower brain-stem we refrained from dissecting completely the adherent capsule and performed immediate tracheostomy before extubation.

The importance of early diagnosis should be stressed. Hemiatrophy of the tongue seems to have for this type of tumour a diagnostic import- ance similar to that attributed to progressive deafness and impaired caloric vestibular reactions for acoustic neuromas.

\section{REFERENCES}

Ignelzi, R. J., and Bucy, P. C. (1967). Intracranial hypoglossal neurofibroma. Case report. Journal of Neurosurgery, 26 , 352-356.

Kirdani, M. A. (1967). The normal hypoglossal canal. American Journal of Roentgenology, Radium Therapy and Nuclear Medicine, 99, 700-704.

McGrew, E. A. (1955). Presentation of case. Journal of American Medical Association, 158, 183-184.

Morelli, R. J. (1966). Intracranial neurilemomma of the hypoglossal nerve. Review and case report. Neurology (Minneapolis), 16, 709-713.

Raney, R. B., Raney, A. A., and Bayless, A. E. (1954). Tumors of hypoglossal nerve; report of case. Bulletin of Los Angeles Neurological Society, 19, 160-165.

Scott, M., and Wycis, H. T. (1949). Intracranial neurinoma of the hypoglossal nerve. Successful removal, case report. Journal of Neurosurgery, 6, 333-336.

Valvassori, G. E., and Kirdani, M. A. (1967). The abnormal hypoglossal canal. American Journal of Roentgenology, Radium Therapy and Nuclear Medicine, 99, 705-711.

Williams, J. M., and Fox, J. L. (1962). Neurinoma of the intracranial portion of the hypoglossal nerve. Review and case report. Journal of Neurosurgery, 19, 248-250. 